\title{
Cross-Layer Optimized Congestion, Contention and Power Control in Wireless Ad Hoc Networks
}

\author{
Eren Gürses* \\ Centre for Quantifiable QoS in Communication Systems ${ }^{\star \star}$ \\ Norwegian University of Science and Technology, \\ O.S. Bragstads Plass, 2E, N-7491 Trondheim, Norway \\ gurses@q2s.ntnu.no
}

\begin{abstract}
Despite some scepticism, cross-layer optimization has largely been accepted as an indispensable part of protocol design for wireless networks. In this respect, generalized network utility maximization (GNUM) has become a widely employed systematic approach for understanding the interconnections between layers and designing efficient cross-layer protocols. In this paper we adopt the GNUM approach and propose a cross-layer optimized congestion, contention and power control algorithm for transport, MAC (medium access control) and physical layers respectively. First we develop an abstract MAC layer model to capture the effects of multiple access in a wireless medium, then express the effective link capacities by relating physical layer capacities to the MAC layer model through average interference. Secondly we construct the GNUM based congestion, contention and power control problem, and devise a primal-based distributed algorithm to solve it. Results show that distributed algorithm obtains very similar results with the centralized one.
\end{abstract}

\section{Introduction}

Following the seminal paper of Kelly et.al. [1], network utility maximization (NUM) has been a versatile tool for understanding, reverse engineering and designing Internet congestion control protocols. Lately starting with [7], a generalized form of NUM has been adapted to wireless networks and widely used in order to expose the interconnections between layers and design alternative layering (or cross-layering) schemes in general [2]. The generalized network utility maximization (GNUM) puts the sum of source utilities and other application imposed needs to the objective function to maximize while relating the other protocol layer parameters through constraints. Source utilities are functions of source rates and different forms of them represent different measures of fairness.

In many works following [7, different cross-layer designs based on the GNUM have been proposed. Here, we only discuss the relevant works from this literature which has a special focus on transport, MAC (medium access control) and

\footnotetext{
* Author has been affiliated with David R. Cheriton School of Computer Science, University of Waterloo, ON, Canada since Jan 2008. E-mail: egurses@uwaterloo.ca

** Centre for Quantifiable QoS in Comm. Systems, CoE, is appointed by The Research Council of Norway, and funded by the Research Council, NTNU and Uninett.
} 
physical layers. The joint congestion-contention problem is addressed in [3], 4], [5] and [6]. In [3] a link scheduling algorithm based on deterministic approximations is proposed. However this algorithm entails a continuous messaging overhead to conduct link scheduling and can not be a replacement of standard simple distributed coordination function (DCF) of 802.11 [9]. Furthermore, in [6] it has been shown that deterministic approximations can not accurately model random access-based MAC. In 4, 5] very similar joint congestion-contention control problems are addressed. However, MAC models used in these works assume saturation conditions for the operation which relies on the existence of at least one packet in MAC layer transmit buffer. This assumption makes the MAC layer model identical to slotted Aloha [10]. In contrast to this, we allow less restrictive non-saturation conditions in the MAC layer operation which is practically the case if transport layer is adapting its rate in coordination with the MAC layer. Actually in our work, link access probabilities regulates the flow to the MAC buffer which prevent saturation mode operation. In 8] a joint power scheduling (power adaptation and link scheduling) and congestion control problem is addressed. They develop a probabilistic model and propose a joint power scheduling and congestion control algorithm using stochastic subgradient method. However the resulting algorithm has the similar disadvantages of [3. On the other hand [7] addressed the problem of joint congestion control and power adaptation. Although the work claims the use of CDMA based MAC layer, the convex optimization process is completely relying on exclusive scheduling of closeby nodes in order to achieve high SIR (signal-to-interference-ratio). However neither an exclusive scheduling algorithm nor its impact on effective link capacities is discussed.

In this paper we extend the congestion control and power adaptation framework in [7] to include the effect of MAC layer. However, integrating the effect of MAC layer to the framework in [7] leads to a set of difficulties like non-convexity and non-separability and necessitates a completely different approach to overcome them. The contributions of this paper start at this point and are given as follows. First we develop an abstract MAC layer model to represent the nonsaturation mode of operation through successful channel access probabilities, $a_{l}$. Second we describe physical layer link capacities $c(P, a)$, based on average interference model in order to consider the effect of MAC layer and then construct the resulting convex GNUM problem. Finally we propose a cross-layer optimized distributed congestion, contention and power control algorithm using primal-based techniques.

The rest of the paper is organized as follows. In Section 2, we first present the system model including the contributions on MAC and physical layer and then construct the GNUM problem for optimal congestion, contention and power control. Next after in Section 3, we first convert the given NUM problem to a convex one and then introduce the distributed primal-based subgradient projection algorithm to solve this convex but non-separable problem. Finally, the simulation results are presented in Section 4 and followed Section 5 where we conclude the paper and give an outlook of possible future work. 


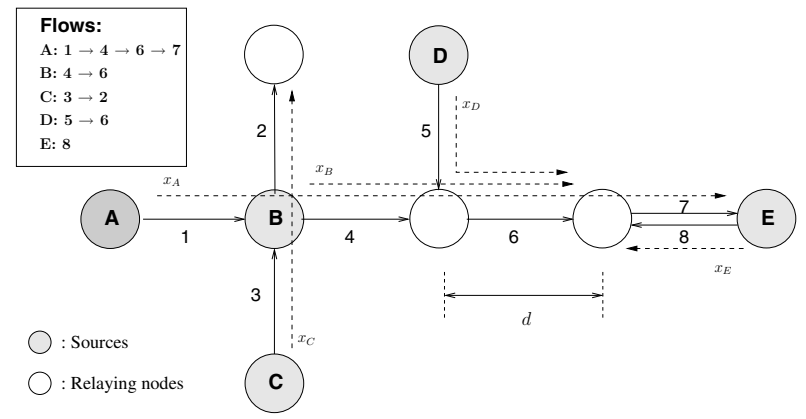

Fig. 1. A sample wireless ad hoc network topology

\section{System Model}

We consider a wireless ad hoc network composed of $N$ nodes and $L$ links where each link is unidirectional and only a number of $S \leq N$ nodes are acting as traffic sources. The set of all links and all sources are denoted as $\mathcal{L}$ and $\mathcal{S}$ respectively. Packets from sources are routed to their destinations according to the established routes which are assumed to be fixed by the network layer at the time of optimization. In our network model the routing matrix $R$ of size $L \times S$ corresponds to these established routes where entries $r_{l s}$ of this matrix take the value of 1 if source $s$ is using link $l$, and 0 otherwise.

In the following subsections, we first introduce the parameters and necessary notations on transport, MAC and physical layers, then present the resulting GNUM formulation.

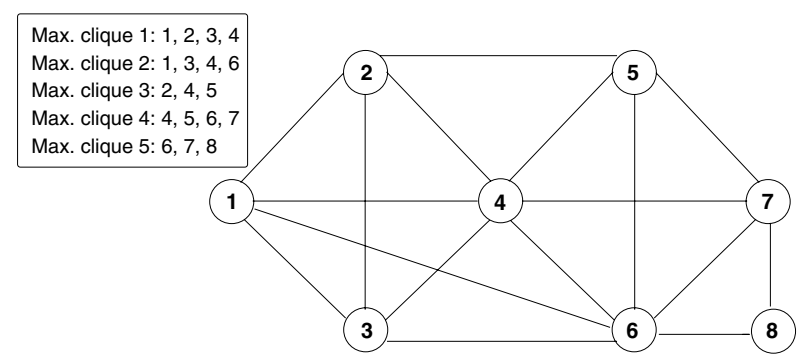

Fig. 2. Conflict graph corresponding to the topology

\subsection{Transport, MAC and Physical Layer Parameters}

The main functionality of the transport layer is to control the end-to-end congestion in a network while maximizing the individual source rates $x_{s}$ of nodes $s \in \mathcal{S}$ according to a given fairness criteria. Congestion control is simply done by controlling $x_{s}$ in order to keep the aggregate flows on links $l \in \mathcal{L}$ less than 
or equal to the effective link capacities observed above MAC layer, while at the same time maximizing the rate of each individual source, generally through a utility function $U_{s}\left(x_{s}\right)$ where different forms of it correspond to different fairness policies. This constrained optimization problem is modeled as a NUM problem and it was recently shown that variants of TCP, together with AQM (Active Queue Management) techniques, are designed to approximately solve it for different forms of utility functions $U_{s}\left(x_{s}\right)$. In classical Internet congestion control problem, NUM formulation is limited to the case of fixed link capacities. However in wireless networks, effective link capacities are functions of MAC and physical layer parameters, and these parameters should be optimized to get a higher network performance. In the rest of this section, we introduce the MAC and physical layer parameters first and then introduce the new generalized network utility maximization (GNUM) problem.

In wireless networks, wireless links implement MAC protocols to have multiple access to the shared medium. In order to capture this behaviour, first we construct an abstract MAC layer model by using conflict graphs. However constructing the conflict graph of a given topology requires the MAC protocol to be specified. Therefore, in the rest of the paper we assume contention based CSMA/CA (carrier sense multiple access with collision avoidance) protocol is employed at the MAC layer. This protocol solves the hidden and exposed terminal problems via a reservation scheme (RTS/CTS) and as a result links within a 2-hop neighborhood contend to capture the medium and if successful, exclusively use it during their transmission. This MAC layer operation enables the links to operate in high SINR region since high interference caused by the simultaneous use of nearby links (i.e. within the 2-hop neighborhood) is eliminated. Later it is shown that this feature is crucial in terms of the convexity of the resulting optimization problem. In the given topology Fig. 1, we assume a separation of $d$ between all neighboring nodes. Assuming that the conflict region of a transmitter is limited to $d$ and the hidden/exposed terminal problems are eliminated by the MAC layer, we can draw the conflict graph in Fig. 2 which helps to visualize the contending links in the network. Note that this graph is not required by the algorithm proposed in Section 3. Only local contention information (within 2-hops distance) is required. In the conflict graph, we label each wireless link of a given topology (Fig (1) with a unique number and represent it as a link node. Each line connecting the two link nodes in the conflict graph means these two links are not allowed to be active at the same time by the CSMA/CA (due to the high mutual interference). All link nodes that are connected to each other form a subgraph that is referred to as a clique. A maximal clique is a complete subgraph that is not contained in any other complete subgraph. Hence the list of maximal cliques for the topology is given as $\{\{1,2,3,4\},\{1,3,4,6\},\{2,4,5\}$, $\{4,5,6,7\},\{6,7,8\}\}$. We define $a_{l}$ as the MAC layer parameter which determines the successful medium access probability on link $l$ after taking the effect of collisions into account. This parameter can be interpreted as the probability (or percentage of time) that link $l$ captures the medium for transmission among the other contending links within a maximal clique $m$. We define a conflict matrix 
$F$ of dimension $M \times L$ which describes how the different wireless links are contending for the channel where $M$ and $L$ denote the total number of maximal cliques and links in the network respectively. The entries $f_{m l}$ of conflict matrix $F$ take the value of 1 for all maximal cliques that the link $l$ is involved and 0 otherwise. For each maximal clique $m$, the sum of medium access probabilities of links that conflict should satisfy the inequality $\sum_{l} f_{m l} a_{l} \leq \epsilon_{m}$, where $\epsilon_{m} \in[0,1]$ denotes the usable portion of a channel after excluding the effect of collisions and interframe spacing (DIFS). The value of $\epsilon_{m}$ is depending on the number of contending links in the max. clique $m$ and can be set adaptively using the results in [10. $\epsilon_{m}=1$ corresponds to the case of perfect scheduling where there is no collision. After running the distributed 2-hop maximum clique identification algorithm, each link $l$ knows the set of links that can not be active at the same time with itself and denote it as $\mathcal{I}^{\prime}(l)$. Then, the links excluded by $\mathcal{I}^{\prime}(l)$ can interfere with $l$ and is given by $\mathcal{I}(l)=\mathcal{L} \backslash \mathcal{I}^{\prime}(l)$. For example if we consider $l=1$, by using the list of maximal cliques in Fig. 2 , every link excluded by $\mathcal{I}^{\prime}(1)=\{1,2,3,4,6\}$ can interfere with $l=1$ (i.e. $\mathcal{I}(1)=\{5,7,8\}$ ).

In interference-limited wireless networks, we can write the physical layer link capacities as $c_{l}(P)=W \log \left(1+K \operatorname{SINR}_{l}(P)\right)$ for a large family of modulations, where $P$ is the transmit power, $W$ is the bandwidth, $\operatorname{SINR}_{l}(P)=$ $G_{l l} P_{l} /\left(\sum_{k \neq l} G_{k l} P_{k}+n_{l}\right)$ is the signal-to-interference-plus-noise-ratio and $K=$ $\left(-\phi_{1}\right) /\left(\log \left(\phi_{2} \mathrm{BER}\right)\right)$ is a constant defined as functions of required bit error rate BER and other constants $\phi_{1}, \phi_{2}$ depending on the modulation. However the expression $c_{l}(P)$ for link $l$ does not consider the effect of MAC layer duty cycle of the interfering links $k \neq l$. Considering the effect of the MAC layer, we say only links $k \in \mathcal{I}(l)$ can be active together with $l$ and interfere. Since an interfering link $k$ can be active only with probability $a_{k}$, then we conclude that $l$ observes an average interference power level of $Q_{k}=a_{k} P_{k}$. Hence we can modify the standard SINR in order to obtain the new signal-to-average interference-plus-noise-ratio $\overline{\operatorname{SINR}}_{l}(P, a)$ in (1) which takes the effect of MAC layer into account.

$$
\overline{\operatorname{SINR}}_{l}(P, a)=\frac{G_{l l} P_{l}}{\sum_{k \in \mathcal{I}(l)} G_{k l} a_{k} P_{k}+n_{l}}
$$

Then by taking the effect of MAC layer into account, physical layer link capacities can be similarly written as $\bar{c}_{l}(P, a)=W \log \left(1+K \overline{\operatorname{SINR}}_{l}(P, a)\right)$. However using this expression, we can safely say that high $\overline{\mathrm{SINR}}_{l}$ can always be maintained since CSMA/CA based MAC layer prevents the nearby (i.e. 2-hop) nodes to operate at the same time. High $\overline{\mathrm{SINR}}_{l}$ allows us to approximate $\bar{c}_{l}(P, a)$ as below, where this function is later shown to be concave in (14) after proper change of variables.

$$
\bar{c}_{l}(P, a) \approx W \log \left(K \overline{\operatorname{SINR}}_{l}(P, a)\right)
$$

\subsection{Generalized Network Utility Maximization Formulation}

Given the protocol layer parameters, GNUM (Generalized Network Utility Maximization) framework is a systematic way of modeling the operation of network 
as a whole through these parameters. In its general form, GNUM appears as a non-linear constrained optimization problem. Similar to NUM, it maximizes the sum of source utilities at the objective function while relating the all protocol layer parameters through the constraints.

The GNUM formulation given in (3)-(7) ties the transport, MAC and physical layer parameters together. The solution gives the optimal transport layer source rates $x_{s}$, MAC layer channel access probabilities $a_{l}$ and physical layer power levels $P_{l}$ that maximize the total network utility. The network utility given as the objective function of (3) corresponds to the sum of individual utilities $U_{s}\left(x_{s}\right)$ of each source $s$ which simply describes the flow fairness criteria in the network. In the rest of the paper we will use proportional fairness by setting the utility function to $U_{s}\left(x_{s}\right)=\log x_{s}$. The values that $x_{s}, a_{l}$ and $P_{l}$ can take are defined by the constraints in (6) -(17). The first constraint in (44) concerns all three layers to be optimized but essentially states that traffic traversing wireless links is bounded by the amount of bandwidth each link actually can maintain to the layers above MAC. The second constraint in (5) describes the link access probability constraint imposed by the MAC layer as given in detail in Section 2.1.

$$
\begin{array}{lll}
\max _{x, a, P} & \sum_{s} U_{s}\left(x_{s}\right) & \\
\text { s. t. } & \sum_{s} r_{l s} x_{s} \leq a_{l} \bar{c}_{l}(P, a) & , \forall l \in \mathcal{L} \\
& \sum_{l} f_{m, l} a_{l} \leq \epsilon_{m} & , \forall m \in \mathcal{F} \\
& 0 \leq a_{l} \leq 1 ; \quad P_{\min } \leq P_{l} \leq P_{\max } & , \forall l \in \mathcal{L} \\
& x_{\min } \leq x_{s} \leq x_{\max } & , \forall s \in \mathcal{S}
\end{array}
$$

\section{Optimal Congestion, Medium Access and Transmit Power Control}

Layering as Optimization Decomposition (LOD) 2] is a popular approach for systematically and distributively solving the cross-layer optimization problems in the form of GNUM. It simply decomposes the optimization problem into subproblems using dual decomposition and assign them to different layers. Each subproblem is solved separately by the assigned layer where the subproblems are coordinated among the layers through Lagrange dual variables. Two necessary conditions for applying LOD are convexity and separability. Separability enables the feasibility of dual-based solutions, whereas the convexity implies the identity of this dual solution with the primal one.

However the given GNUM formulation in (3)-(7) is neither convex and nor separable in its dual. Due to its non-separable nature over $a$ and $P$, we focus on primal-based algorithms. Nevertheless, convexity is required by primal-based algorithms to guarantee the global maximum since any local optimum is necessarily the global one in a convex optimization problem. Converting the original optimization problem into a convex one is achieved by change of variables 
$x_{s}=e^{\hat{x}_{s}}, a_{l}=e^{\hat{a}_{l}}, P_{l}=e^{\hat{P}_{l}}$. Defining $Q_{l}=e^{\hat{Q}_{l}}$ and using the expression for average power $Q_{l}=a_{l} P_{l}$ we obtain $\hat{Q}_{l}=\hat{a}_{l}+\hat{P}_{l}$. Replacing $\hat{a}_{l}=\hat{Q}_{l}-\hat{P}_{l}$ we conclude that the following optimization problem over $\hat{x}, \hat{Q}, \hat{P}$ is convex, if both $U_{s}^{\prime}\left(\hat{x}_{s}\right)$ and $\bar{c}_{l}^{\prime}(\hat{Q}, \hat{P})$ is concave [11.

$$
\begin{array}{lll}
\max _{\hat{x}, \hat{Q}, \hat{P}} & \sum_{s} U_{s}^{\prime}\left(\hat{x}_{s}\right) & \\
\text { s. t. } & \sum_{s} r_{l s} e^{\hat{x}_{s}-\hat{Q}_{l}+\hat{P}_{l}} \leq \bar{c}_{l}^{\prime}(\hat{Q}, \hat{P}) & , \forall l \in \mathcal{L} \\
& \sum_{l} f_{m, l} e^{\hat{Q}_{l}-\hat{P}_{l}} \leq \epsilon_{m} & , \forall m \in \mathcal{F} \\
& \hat{Q}_{\min } \leq \hat{Q}_{l} \leq \hat{Q}_{\max } ; \quad \hat{P}_{\min } \leq \hat{P}_{l} \leq \hat{P}_{\max } & , \forall l \in \mathcal{L} \\
& \hat{x}_{\min } \leq \hat{x}_{s} \leq \hat{x}_{\max } & , \forall s \in \mathcal{S}
\end{array}
$$

The new utility function obtained as $U_{s}^{\prime}\left(\hat{x}_{s}\right)=\hat{x}_{s}$ is concave. On the other hand, we change the variables $a_{l}=e^{\hat{Q}_{l}-\hat{P}_{l}}$ and $P_{l}=e^{\hat{P}_{l}}$ in (11) and (2). Then the new link SINR is obtained as (13) after setting $\overline{\operatorname{SINR}}_{l}^{\prime}(\hat{P}, \hat{Q})=\overline{\operatorname{SINR}}_{l}\left(e^{\hat{P}}, e^{\hat{Q}-\hat{P}}\right)$.

$$
\overline{\operatorname{SINR}}_{l}^{\prime}(\hat{P}, \hat{Q})=\frac{G_{l l} e^{\hat{P}_{l}}}{\sum_{k \in \mathcal{I}(l)} G_{k l} e^{\hat{Q}_{k}}+n_{l}}
$$

Similarly, new physical layer link capacities are given by (14) after setting $\bar{c}_{l}^{\prime}(\hat{Q}, \hat{P})=\bar{c}_{l}\left(e^{\hat{P}}, e^{\hat{Q}-\hat{P}}\right)$ and using (13). Note that this is a concave function of $\hat{P}$ and $\hat{Q}$, since log of exponentials is convex[11]. Hence the problem in (8)(12) is convex.

$$
\bar{c}_{l}^{\prime}(\hat{Q}, \hat{P})=\frac{1}{K_{\max }}\left[\log \left(K G_{l l}\right)+\hat{P}_{l}-\log \left(\sum_{k \in \mathcal{I}(l)} G_{k l} e^{\hat{Q}_{k}}+n_{l}\right)\right]
$$

\subsection{Primal-Based Algorithm}

We propose a primal-based algorithm for the distributed solution of the nonseparable convex optimization problem in (8)-(12). For this purpose, we write rate and contention constraints in (9) and (10) in the standard form $h_{l}(\hat{x}, \hat{Q}, \hat{P}) \leq$ 0 and $w_{m}(\hat{Q}, \hat{P}) \leq 0$ respectively where $h_{l}(\hat{x}, \hat{Q}, \hat{P})$ and $w_{m}(\hat{Q}, \hat{P})$ are given as:

$$
\begin{gathered}
h_{l}(\hat{x}, \hat{Q}, \hat{P}) \triangleq \sum_{s} r_{l s} e^{\hat{x}_{s}-\hat{Q}_{l}+\hat{P}_{l}}-\bar{c}_{l}^{\prime}(\hat{Q}, \hat{P}) \\
w_{m}(\hat{Q}, \hat{P}) \triangleq \sum_{l} f_{m l} e^{\hat{Q}_{l}-\hat{P}_{l}}-\epsilon_{m}
\end{gathered}
$$

Then, by using penalty function method the new objective function $V(\hat{x}, \hat{Q}, \hat{P})$ is obtained as follows where $\kappa$ is a positive constant [11].

$$
V(\hat{x}, \hat{Q}, \hat{P})=\sum_{s} U_{s}^{\prime}\left(\hat{x}_{s}\right)-\kappa \sum_{l} \max \left\{0, h_{l}(\hat{x}, \hat{Q}, \hat{P})\right\}-\kappa \sum_{m} \max \left\{0, w_{m}(\hat{Q}, \hat{P})\right\}
$$


Hence, instead of the original one, the following optimization problem can now alternatively be solved over the primal variables.

$$
\begin{array}{rll}
\max _{\hat{x}, \hat{Q}, \hat{P}} & V(\hat{x}, \hat{Q}, \hat{P}) & \\
\text { s. t. } & \hat{Q}_{\min } \leq \hat{Q}_{l} \leq \hat{Q}_{\max } & , \forall l \in \mathcal{L} \\
& \hat{x}_{\min } \leq \hat{x}_{s} \leq \hat{x}_{\max } & , \forall s \in \mathcal{S} \\
& \hat{P}_{\min } \leq \hat{P}_{l} \leq \hat{P}_{\max } & , \forall l \in \mathcal{L}
\end{array}
$$

Since $V(\hat{x}, \hat{Q}, \hat{P})$ is not differentiable, the primal-based solution for the problem in (18) can be distributively obtained by using subgradient projection algorithm given by (19)-(21). Subgradients are later described in (24)-(26). Each source $s$ and link $l$ respectively use the equations (19) and (20)-(21) to update their primal parameters at each iteration. And since the problem is convex, any local optimum obtained by this algorithm is guaranteed to be the global one.

$$
\begin{aligned}
& \hat{x}_{s}(t+1)=\left[\hat{x}_{s}(t)+\beta(t) g_{\hat{x}_{s}}\left(\hat{x}_{s}, \hat{Q}_{l}, \hat{P}_{l}\right) \mid \begin{array}{l}
\hat{x}_{s}=\hat{x}_{s}(t), \\
\hat{Q}_{l}=\hat{Q}_{l}(t), \\
\hat{P}_{l}=\hat{P}_{l}(t)
\end{array}\right]_{\hat{x}_{\min }}^{\hat{x}_{\max }} \\
& \hat{Q}_{l}(t+1)=\left[\hat{Q}_{l}(t)+\beta(t) g_{\hat{Q}_{l}}\left(\hat{x}_{s}, \hat{Q}_{l}, \hat{P}_{l}\right) \begin{array}{l}
\hat{x}_{s}=\hat{x}_{s}(t), \\
\hat{Q}_{l}=\hat{Q}_{l}(t), \\
\hat{P}_{l}=\hat{P}_{l}(t)
\end{array}\right]_{\hat{Q}_{\min }}^{\hat{Q}_{\max }} \\
& \hat{P}_{l}(t+1)=\left[\hat{P}_{l}(t)+\beta(t) g_{\hat{P}_{l}}\left(\hat{x}_{s}, \hat{Q}_{l}, \hat{P}_{l}\right) \begin{array}{l}
\hat{x}_{s}=\hat{x}_{s}(t), \\
\hat{Q}_{l}=\hat{Q}_{l}(t), \\
\hat{P}_{l}=\hat{P}_{l}(t)
\end{array}\right]_{\hat{P}_{\min }}^{\hat{P}_{\max }}
\end{aligned}
$$

First we define the functions $\gamma_{m}$ and $\delta_{l}$ in (22)-23) which can be respectively interpreted as the indication of high contention in max. clique $m$ and congestion on link $l$.

$$
\begin{gathered}
\gamma_{m}= \begin{cases}0 & , \text { for } \sum_{l} f_{m l} e^{\hat{Q}_{s}-\hat{P}_{l}} \leq \epsilon_{m} \\
1 & , \text { otherwise }\end{cases} \\
\delta_{l}= \begin{cases}0 & , \text { for } \sum_{s} r_{l s} e^{\hat{x}_{s}-\hat{Q}_{l}+\hat{P}_{l}} \leq \bar{c}_{l}^{\prime}(\hat{Q}, \hat{P}) \\
1 & , \text { otherwise }\end{cases}
\end{gathered}
$$

After defining $\gamma_{m}$ and $\delta_{l}$ it is easy to calculate subgradients. However for the sake of brevity, we denote the common terms as $D_{1}\left(\hat{Q}_{l}, \hat{P}_{l}\right) \triangleq e^{\hat{Q}_{l}-\hat{P}_{l}} \sum_{m} f_{m l} \gamma_{m}$ and $D_{2}\left(\hat{x}_{s}, \hat{Q}_{l}, \hat{P}_{l}\right) \triangleq \delta_{l} e^{-\hat{Q}_{l}+\hat{P}_{l}} \sum_{s} r_{l s} e^{\hat{x}_{s}}$ where finally the subgradients are given by: 


$$
\begin{aligned}
& g_{\hat{x}_{s}}\left(\hat{x}_{s}, \hat{Q}_{l}, \hat{P}_{l}\right)=1-\kappa e^{\hat{x}_{s}} \sum_{l} \delta_{l} r_{l s} e^{-\hat{Q}_{l}+\hat{P}_{l}} \\
& g_{\hat{P}_{l}}\left(\hat{x}_{s}, \hat{Q}_{l}, \hat{P}_{l}\right)=\kappa\left[D_{1}\left(\hat{Q}_{l}, \hat{P}_{l}\right)-D_{2}\left(\hat{x}_{s}, \hat{Q}_{l}, \hat{P}_{l}\right)+\frac{\delta_{l}}{K_{\max }}\right] \\
& g_{\hat{Q}_{l}}\left(\hat{x}_{s}, \hat{Q}_{l}, \hat{P}_{l}\right)=\kappa\left[D_{2}\left(\hat{x}_{s}, \hat{Q}_{l}, \hat{P}_{l}\right)-D_{1}\left(\hat{Q}_{l}, \hat{P}_{l}\right)-\frac{e^{\hat{Q}_{l}}}{K_{\max }} \sum_{j \in \mathcal{I}(l)} \frac{\delta_{j} G_{l j}}{e^{\hat{P}_{j}} G_{j j}} \overline{\operatorname{SINR}}_{j}^{\prime}\left(\hat{P}_{j}, \hat{Q}\right)\right]
\end{aligned}
$$

In the given subgradient projection algorithm, positive subgradients correspond to an increase in the related primal variables. $D_{1}$ is a contention related term. If there is high contention in any of the max. cliques that link $l$ belongs to, then $D_{1}$ leads to an increase in $\hat{P}_{l}$ and decrease in $\hat{a}_{l}$ through $\hat{Q}_{l}$ as respectively given by subgradients in (25) and (26). On the other hand a non zero $D_{2}$ indicates congestion on link $l$. Then $D_{2}$ leads to a decrease in $\hat{P}_{l}$ (25) and increase in $\hat{a}_{l}$ through $\hat{Q}_{l}$ on link $l$ according to (25) and (26) respectively. Besides $D_{1}$ and $D_{2}$, a congested link increases its power $\hat{P}_{l}$ due to the term $\delta_{l} / K_{\max }$ in (25). Similarly considering the last term of (26), in case of congestion on a mutually interfering link $j \in \mathcal{I}(l)$ with link $l, \hat{a}_{l}$ is decreased in order to reduce the interference on that remote link $j$ by reducing $\hat{Q}_{l}$. As a result, reducing interference on $j$ necessarily increases $\mathrm{SINR}_{j}$ and physical layer link capacity $\bar{c}_{j}^{\prime}(P, a)$. The reader should notice that due to this last term, the update of (26) requires exchange of the message $\overline{\operatorname{SINR}}_{j}^{\prime}\left(\hat{P}_{j}, \hat{Q}\right) G_{l j} / e^{\hat{P}_{j}} G_{j j}$ from remotely located, interfering and congested link $j$ to $l$ as given in (26). However as the algorithm converges the number of congested links in the network will reduce and the amount of message exchange will steadily decrease.

\section{Simulation and Discussion}

In our simulations, we use the simple ad hoc wireless multi-hop network topology in Figure. 1 to illustrate the optimal congestion, contention and power control algorithm. Although we consider a simple topology for the ease of showing the optimization variables, it should be noted that the proposed algorithm is fully distributed and yet scalable. The given topology is composed of $N=8$ nodes, $L=8$ links, $S=5$ sources and $M=5$ max. cliques where the separation between all neighboring nodes is $50 \mathrm{~m}$. According to the performance analysis of 802.11, we select $\epsilon_{m}=0.85$ due to collisions and interframe spacing. We set BER $=10^{-3}, \phi_{1}=\phi_{2}=1, W=5 \mathrm{MHz}$ and $G_{l k}=d_{l k}^{-4}$ where $d_{l k}$ is the distance between transmitter of link $l$ and receiver of link $k$. Then we determine the noise level $n_{l}$ on each link by assuming an $8 \mathrm{~dB}$ SNR level when transmitter power is set to $2.5 \mathrm{~mW}$. We normalize link capacities in (2) with $W K_{\max }$ where $K_{\max }=4$. We set $\left(x_{\min }, x_{\max }\right)=(0,1)$ after the normalization and $\left(P_{\min }, P_{\max }\right)=(0.8,28) \mathrm{mW}$. Finally we set the parameters related to algorithm as $\kappa=4$ and $\beta(t)=0.01$.

Results of the proposed optimal congestion, contention and power control algorithm is given in Fig. 3. It is first observable that flows requiring less resources 


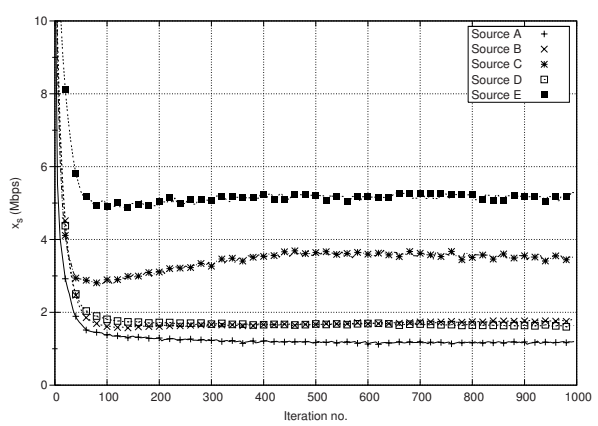

(a) Congestion control

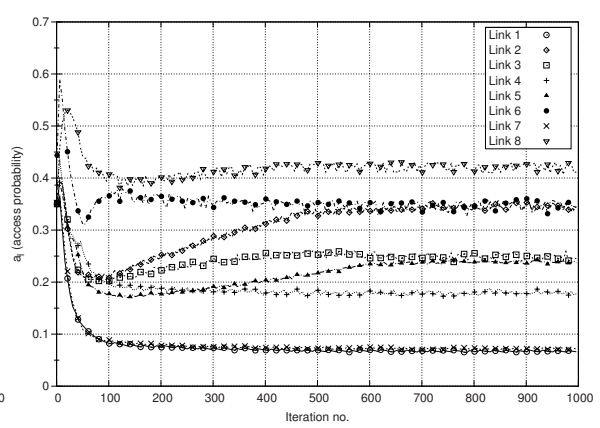

(b) Contention control

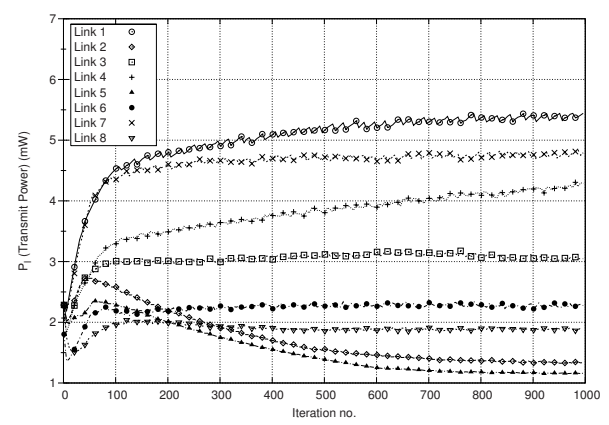

(c) Power adaptation

Fig. 3. Primal variables that describe (a) transport layer rates $x_{s}$, (b) MAC layer link access probabilities $a_{l}$, (c) physical layer transmit power levels $P_{l}$

from the network (for. ex. number of hops) are getting higher bandwidth. Furthermore we can also observe that source rates $x_{s}$ are converging very fast where the other primal variables need more iterations to converge. However besides these observations, it is hard to understand how $a_{l}$ and $P_{l}$ are optimal (i.e. maximizing network utility) since the problem is not separable over them. Therefore rather than examining $a_{l}$ and $P_{l}$ separately, we investigate the effective capacity observed above MAC layer $a_{l} \bar{c}_{l}(P, a)$ which is a function of both $a_{l}$ and $P_{l}$. These effective capacities $a_{l} \bar{c}_{l}(P, a)$ are plotted in Fig. 4 (b) and can be calculated by using $a_{l}$ in Fig. 3(b) and $\bar{c}_{l}(P, a)$ in Fig. $4(\mathrm{a})$. In the following discussion we denote any link $k \in\{1, \ldots, 8\}$ as $\mathrm{L} k$ in short. From the topology given in Fig. 1 we can see that L6 (short notation for link 6) is used by 3 flows, which implies increasing the effective capacity on L6 may lead to a rate increase in 3 flows $x_{A}$, $x_{B}$ and $x_{D}$. Although L4 carries 2 flows (second most used link), these flows $x_{A}$ and $x_{B}$ use the link L6 as well, hence capacity of L4 is bounded by the effective capacity of L6. On the other hand L8 is used by a single flow $x_{E}$, and this flow is not using any other link. Then any increase in effective capacity of L8 directly results in an increase in the utility of $x_{E}$. Hence effective capacities on L6 and L8 are the most important ones for maximizing the network utility. Therefore 

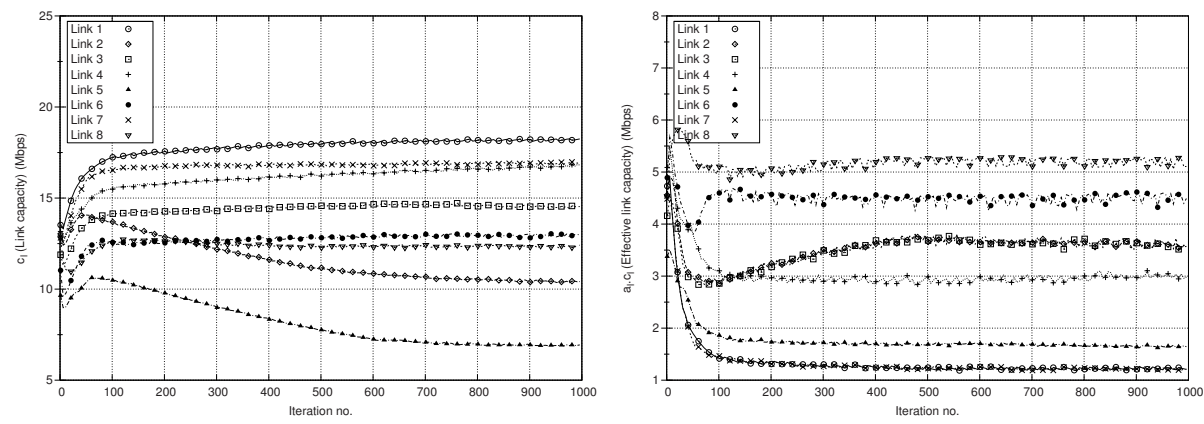

Fig. 4. (a) Physical layer link capacities $\bar{c}_{l}(P, a)$, (b) effective link capacities $a_{l} \cdot \bar{c}_{l}(P, a)$

these two most important links L6 and L8 get the highest effective link capacity in Fig. 4(b). On the other hand effective capacities of L1 and L7 are the least important ones in terms of their contribution to the network utility, since the only flow $x_{A}$ using them also uses L4 and L6. Hence the effective capacities of L1 and L7 are bounded by L4 and L6. Therefore in Fig. 4(b) they acquire the lowest values for effective link capacities. Finally, L2 and L3 are used only by the single flow $x_{C}$ which couples the effective capacities of these links. Since any increase on these link capacities excluding the other, do not contribute to the utility of $x_{C}$. This coupled behaviour is also clearly observable in Fig. 4(b) where the values of effective link capacities follow each other.

Finally we compare the results of the distributed algorithm with the results of MATLAB's fmincon(.) which solves (8)-(12) in a centralized way. However fmincon returns the maximum possible values for primal variables eventhough they do not increase the objective function. Therefore the optimal power levels $P_{l}^{*}$ for $L 1$ and $L 7$ are significantly different from results of the distributed algorithm. However the remaining results are conforming with the results of the distributed algorithm (with some error).

Table 1. Results of the centralized solution obtained by fmincon(.)

\begin{tabular}{|c|c|c|c|c|c|}
\hline Sources & A & B & $\mathrm{C}$ & D & $\mathrm{E}$ \\
\hline$x_{s}^{*}$ & 1.2375 & 1.6570 & 3.4787 & 2.0328 & 5.0031 \\
\hline
\end{tabular}

\begin{tabular}{c||ccccccccc} 
Links & 1 & 2 & 3 & 4 & 5 & 6 & 7 & 8 & \\
\hline$a_{l}^{*}$ & 0.0534 & 0.3911 & 0.2388 & 0.1666 & 0.2364 & 0.3911 & 0.0559 & 0.4030 & \\
$P_{l}^{*}$ & 14.9776 & 0.9992 & 3.3494 & 4.8014 & 1.8542 & 2.0454 & 14.3182 & 1.9851 & $(\mathrm{~mW})$ \\
$a_{l}^{*} \cdot c_{l}^{*}$ & 1.0683 & 3.4787 & 3.4787 & 2.8945 & 2.0328 & 4.9273 & 1.1175 & 5.0031 & $(\mathrm{Mbps})$
\end{tabular}

\section{Conclusions and Future Work}

In this paper we formulate the optimal joint congestion, contention and power control problem in wireless ad hoc networks through GNUM. Considering the 
effect of MAC layer on physical layer link capacities, the original GNUM problem become non-convex and non-separable which can not be directly solved by LOD. After converting the problem to a convex one, we devise a primal-based distributed algorithm to solve the given cross-layer optimization problem. Simulation results show that the results of the centralized optimal solution obtained by MATLAB can be reached without any global coordination.

In this work we limit our GNUM model to the basic modes of operation in PHY, MAC and network layers. An immediate step in the future is to evaluate the performance of the proposed algorithm in an actual network setting. Another possible future direction is to extend these basic modes of operation to more complicated ones like PHY layer cooperative diversity, MAC layer multi-channel assignement and network layer optimal routing which could lead to a dramatic increase in the network performance.

\section{References}

1. Kelly, F.P., Maulloo, A., Tan, D.: Rate control for communication networks: Shadow prices, proportional fairness and stability. Journal of Operations Research Society 49(3), 237-252 (1998)

2. Chiang, M., Low, S.H., Calderbank, A.R., Doyle, J.C.: Layering as optimization decomposition: A mathematical theory of network architectures. Proceedings of the IEEE 85(1), 255-312 (2007)

3. Chen, L., et al.: Joint congestion control and media access control design for ad hoc wireless networks. In: Proc. of IEEE INFOCOM, vol. 3, pp. 2212-2222 (2005)

4. Wang, X., et al.: Cross-layer rate control for end-to-end proportional fairness in wireless networks with random access. In: Proc. ACM Mobihoc, pp. 157-168 (2005)

5. Lee, J.-W., Chiang, M., Calderbank, R.A.: Jointly optimal congestion and contention control based on network utility maximization. IEEE Communication Letters 10(3), 216-218 (2006)

6. Lee, J.-W., Chiang, M., Calderbank, R.A.: Utility-optimal medium access control: Reverse and forward engineering. In: Proc. of IEEE INFOCOM, pp. 1-13 (2006)

7. Chiang, M.: Balancing transport and physical layers in wireless multihop networks: Jointly optimal congestion control and power control. IEEE Journal on Selected Areas in Comm. 23(1), 104-116 (2005)

8. Lee, J.-W., Mazumdar, R., Shroff, N.B.: Joint opportunistic power scheduling and end-to-end rate control for wireless ad hoc networks. IEEE Trans. on Vehicular Technology 56(2), 801-808 (2007)

9. IEEE 802.11 WG: International standard for information technology local and metropolitan area networks-specific requirements Part 11: Wireless LAN MAC and PHY specifications (1999)

10. Bianchi, G.: Performance analysis of the IEEE 802.11 distributed coordination function. IEEE Journal on Selected Areas in Comm. 18(3), 535-547 (2000)

11. Boyd, S., Vandenberghe, L.: Convex Optimization. Cambridge University Press, Cambridge (2004) 